\title{
Mechanical and kinematic alignment in total knee arthroplasty
}

\author{
Marco Schiraldi, Giancarlo Bonzanini, Danilo Chirillo, Vito de Tullio \\ Department of Orthopaedics and Traumatology, Regional Center for Joint Arthroplasty, ASO Alessandria, Italy \\ Contributions: (I) Conception and design: All authors; (II) Administrative support: All authors; (III) Provision of study materials or patients: All authors; \\ (IV) Collection and assembly of data: All authors; (V) Data analysis and interpretation: All authors; (VI) Manuscript writing: All authors; (VII) Final \\ approval of manuscript: All authors. \\ Correspondence to: Marco Schiraldi. Department of Orthopaedics and Traumatology, Regional Center for Joint Arthroplasty, ASO Alessandria, Italy. \\ Email: mschiraldi@ospedale.al.it.
}

\begin{abstract}
In the last 10 years many studies have questioned if the strive to mechanical align any knee may pose some problems related to ligament misbalancing that could explain the high rate of disappointed patients, almost $20 \%$ in some reports. Proper indication and difference between patient's and surgeon's expectations are among the most important one's but it must be underlined that, there is indeed a sharp difference between normal knee kinematics, prosthetic knee kinematics and arthritic knee kinematics being the last one extremely variable. A so called kinematic alignment has recently been developed in order to improve patient's knee function and pain control minimizing any surgical gesture focused on ligaments balance. The amount of bone resections may not affect limb alignment but has an important consequence in ligament tension and balance, clinical result and function therefore a measured bone resection technique is essential in order to perform a proper kinematic alignment. Purpose of this paper is to briefly review the different alignment procedures used for TKA and to discuss their definitions, concepts and evidence on outcome.
\end{abstract}

Keywords: Cinematic alignment; mechanical alignement; total knee arthroplasty (TKA)

Submitted Mar 03, 2016. Accepted for publication Mar 15, 2016.

doi: $10.21037 /$ atm.2016.03.31

View this article at: http://dx.doi.org/10.21037/atm.2016.03.31

\section{Introduction}

Restoration of knee alignment is considered one of the mainstays of a successful total knee arthroplasty (TKA) in order to achieve a neutral mechanical axis passing from the center of femoral head thorough the center of the knee joint line to the center of the ankle.

In the last 10 years many studies have questioned if the strive to mechanical align any knee may pose some problems related to ligament misbalancing that could explain the high rate of disappointed patients, almost $20 \%$ in some reports $(1,2)$.

According our opinion unsatisfactory results are related to multifactorial causes.

Proper indication and difference between patient's and surgeon's expectations are among the most important one's but it must be underlined that there is indeed a sharp difference between normal knee kinematics, prosthetic knee kinematics and arthritic knee kinematics being the last one extremely variable.

Paradoxically, an operation performed on an extremely painful patient affected by a severe knee arthritis can be less problematic because that given knee kinematics is already totally gone and that knee will benefit from anything else that will restore sufficient function and motion.

On the contrary, a different surgical behavior and approach must be considered when dealing with the majority of knee arthritis where ligaments imbalance and bone deformity don't affect knee kinematics so deeply.

These patients may be more challenging for the inexperienced surgeon, they only have indeed a very modest alteration of knee kinematics and the consequences of a TKA operation (ligament release, cruciate ligament sacrifice, new alignment) coupled with the new prosthetic kinematic may explain a significant part of unsatisfactory results.

Aiming to mechanical alignment only, may arise 
undesirable kinematic consequences if femoral and tibial components are positioned in such a way that the new joint line is raised and not parallel or perpendicular to the three axes that describe tibiofemoral and patella femoral kinematics.

TKA alignment is a relative concept; indeed there are two separate ideas of alignment: component alignment and limb alignment as a whole and both may strictly interact with each other and may result in the same limb alignment but with a very different knee kinematics, motion and clinical results (3).

A so called kinematic alignment has recently been developed in order to improve patient's knee function and pain control minimizing any surgical gesture focused on ligaments balance (4-6).

The amount of bone resections may not affect limb alignment but has an important consequence in ligament tension and balance, clinical result and function therefore a measured bone resection technique is essential in order to perform a proper kinematic alignment.

The idea of kinematic alignment is not totally new: it is inspired indeed from the concept of anatomical alignment of Hungerford and Krackow (7).

Purpose of this paper is to briefly review the different alignment procedures used for TKA and to discuss their definitions, concepts and evidence on outcome.

\section{Alignment axes}

The vertical axes is a vertical line that in normal AP RX weight bearing goes from the center of the pubic symphysis to the ground (8).

The mechanical axis of the lower limb is a line extended from the center of the femoral head to the center of the ankle and in normal condition it crosses the center of the knee joint (9).

The femoral part of this line that goes from the center of the femoral head to the center of the knee (the intercondylar notch of the distal femur) is called femoral mechanical axis while the distal or tibial part that goes from the center of the tibial proximal epiphysis to the center of the ankle joint is called tibial mechanical axis.

In normal condition the two axes, femoral and tibial one describe a straight angle or more precisely a medial angle slightly less than $180^{\circ}(10,11)$.

The femoral and tibial anatomical axes are identified within the intramedullary bone canal and may be drawn with a line bisecting both the femur and tibia in an one half or, less precisely, drawing a line connecting the center of the femoral or tibial shaft to point $10 \mathrm{~cm}$ above or below the knee joint respectively.

On anteroposterior evaluation the femoral anatomical axis has a 5-7 degree of inclination difference than his mechanic axis while in normal condition the tibial anatomical axis coincides with the mechanical one and as consequence these 2 anatomical axis of femur and tibia describe a lateral angle called femorotibial angle (FTA) whose range depends on sex, height, femoral hip offset, rotation and physical anthropology.

The FTA is approximately $178^{\circ}$ and $175^{\circ}-176^{\circ}$ in Caucasian men and women respectively while slightly less in Asian people but may markedly deviate in case of associate torsional or flexion deformity (12).

\section{Kinematic axis}

Mechanical and anatomical axes are well described on a plain long standing $\mathrm{X}$-ray. The kinematic axes are the 3 axes that describe knee motion (13). 'Kinein' in old Greek means to move and the word kinematics is the part of classical mechanics that describe the motion of points, bodies and system of bodies without consideration of the masses of those objects nor the forces that may have caused the motion.

The three kinematic axes are functional axes of movement about which the knee flexes and rotates. Kinematic alignment is therefore a dynamic and $3 \mathrm{D}$ alignment and cannot be represented but only partially at plain X-ray.

As stated before we have 3 kinematic axes: (I) the transverse axis in the femur about which the tibia flexes and extends located in the center of a circle inscribed in the femoral condyles (13); (II) the transverse axis in the femur about which the patella flexes and extends, parallel to the patellar axis and parallel to the first transverse femoral axis $(5,6)$; and (III) the longitudinal axis in the tibia about which the tibia internally and externally rotates on the femur. The longitudinal axis in the tibia is then perpendicular to the previous 2 axes (8).

\section{Normal knee alignment}

Normal FTA is slightly more valgus in women than men. In general the normal knee joint alignment is $2^{\circ}-3^{\circ}$ of varus compared with the mechanical axis although healthy nonarthritic patients may have different values. In fact obesity, activity and muscle strength, all play a crucial role in the 
development of arthritis also with a perfect aligned knee. Several studies have shown the prevalence of a constitutional varus knee with a significant percentage of valgus morphology moreover even individuals characterized with different alignment between left and right knee $(3,14,15)$.

\section{Total knee arthroplasty (TKA) alignment}

\section{Anatomic alignment}

Purpose of this technique originally described by Hungeford and Krackow (7) is to position the femoral and tibial component in order to anatomically recreate the joint line with the overall component alignment at $2^{\circ}-3^{\circ}$ of varus in relation to the mechanical axis of the lower limb (7).

According this technique it is postulated a normal 6 degrees FTA therefore this angle may be reached with a femoral cut made at approximately $8-9^{\circ}$ of valgus combined with the $2^{\circ}-3^{\circ}$ of varus angulation of the tibial cut. Additionally purpose of this alignment is to provide for a joint line that is parallel to the ground during normal gait (7).

\section{Mechanical alignment}

Described by Insall et al., the mechanical alignment is the widest method used in TKA probably for the high reproducibility and easiness (16). It requires an initial femoral cut that must be perpendicular to the mechanical axis of the femur; the tibial resection must be performed perpendicular to the mechanical axis of the tibia. The result is a knee that is aligned at $4^{\circ}-5^{\circ}$ valgus in general but may be changed according to patient's height and limb morphology. Purpose of this alignment is to create and even load distribution on the new joint line; According to Insall, the knee joint aligned thorough the anatomical alignment may be loaded more medially with a medial tibial plateau fixation failure. He also promoted the femoral component positioning at $3^{\circ}$ of external rotation in order to balance flexion and extension gaps.

As stated before most surgeries and clinical studies are based on mechanical alignment that for this reason must still be considered the so called 'gold standard'. One of the most important studies was conducted by Fang et al. (17): their retrospective study was based on 6,070 TKA looking for survivorship in well aligned knee compared with that of their outliers (more than 3 degrees valgus or varus). They found 51 failures: 21 in the neutral cohort $(0.5 \%)$, 18 in the varus $(1.8 \%)$ and $12(1.5 \%)$ in the valgus group.
They concluded that the goal of TKA should be to restore alignment within $2.4^{\circ}-7.2^{\circ}$ of valgus. Jeffery et al. (18) reported on a much smaller cohort (115 knees). They recorded a $3 \%$ rate loosening when the mechanical axis crossed the middle one third of the prosthesis ( 2 out of 78 TKA) while the loosening rate (24\%) was significantly increased when the mechanical axis was shifted either medially or laterally (9 out of 37 TKA). Ritter reviewed 6,070 knees in 3,992 patients with a minimum of 2 years of follow-up (19). Each knee was classified on the basis of postoperative alignment (overall tibiofemoral alignment and alignment of the tibial and the femoral component in the coronal plane). Failure was most likely to occur if the orientation of the tibial component was $<90^{\circ}$ relative to the tibial axis and the orientation of the femoral component was $\geq 8^{\circ}$ of valgus (failure rate, $8.7 \% ; \mathrm{P}<0.0001$ ). In contrast, failure was least likely to occur if both the tibial and the femoral component were in a neutral orientation $\left(\geq 90^{\circ}\right.$ and $<8^{\circ}$ of valgus, respectively) [failure rate, $0.2 \%$ (9 of 4,633); $\mathrm{P}<0.0001]$. Vanlommel et al. (20) studied a cohort of 132 consecutive patients (143 knees) with pre-operative varus alignment that was evaluated with a mean followup period of 7.2 years. Based upon the post-operative alignment, patients were stratified into three groups: neutral, mild varus, and severe varus. All patients had postoperative improvements in Knee Society Score (KSS). Knees that were left in mild varus scored significantly better for the KSS and the Western Ontario and McMaster Universities Arthritis Index, compared with knees that were corrected to neutral and knees that were left in severe varus exceeding $6^{\circ}$. No revisions occurred in any of the groups at midterm follow-up. Parratte et al. (21) reviewed retrospectively the clinical and radiographic data following 398 primary total knee arthroplasties performed with cement in 280 patients from 1985 to 1990 to determine the 15-year Kaplan-Meier survival rate. At the time of the latest follow-up, 45 (15.4\%) of the 292 implants in the mechanically aligned group (knees with a mechanical axis of $0^{\circ} \pm 3^{\circ}$ ) had been revised for any reason, compared with 14 $(13 \%)$ of the 106 implants in the outlier group (knees with a mechanical axis of beyond $0^{\circ} \pm 3^{\circ}$ ). They concluded that a postoperative mechanical axis of $0^{\circ} \pm 3^{\circ} \mathrm{did}$ not improve the 15 -year implant survival rate following these 398 modern total knee arthroplasties and that describing alignment as a dichotomous variable (aligned $v s$. malaligned) on the basis of a mechanical axis goal of $0^{\circ} \pm 3^{\circ}$ is of little practical value for predicting the durability of modern TKA implants. Recently, two systematic literature reviews were conducted 
to investigate the association between malalignment following primary TKR and revision rates: Hadi et al. (22) concluded that their meta-analysis on the effect of malalignment on revision rates is likely to be modest and interestingly, studies that used mechanical alignment in the coronal plane demonstrated no association with revision rates. Abdel et al. (23) pointed to review the anatomic alignment of the knee, the historical and contemporary data on a neutral mechanical axis in TKR, and the feasibility of kinematically-aligned TKRs. According their literature review, a neutral mechanical axis remains the optimal guide to alignment.

\section{Kinematic alignment}

Despite good alignment a significant part of the patients $(15-20 \%)(1,2)$ are still disappointed with their artificial knee. For this reason several studies have questioned if the classical or mechanical alignment may actually be the right technique in order to create a well-balanced and functioning knee. The development of a kinematic alignment aims to realize a well-balanced artificial joint during the whole arc of motion. The concept of kinematic alignment has gained interest since the description of the different transverse axis of the femur and of the cylindrical shape of the posterior part of the knee condyles: some authors have questioned if the strive to mechanically align the knee may have undesirable kinematic consequences because positioning of the components may change ligaments tension and arise stress on an altered joint line and thus being a source of pain during knee motion and function. The concept of kinematic alignment is to restore normal knee function by aligning the distal and posterior femoral joint line of the femoral component according the functional femoral transverse axes and joint line of the tibial component to those of the normal or pre arthritic status. Alignment of the femoral component with the normal joint line may be reached when the calipered thickness of the distal and posterior femoral resection after correcting for cartilage and bone wear equal the thickness of the distal and posterior femoral component. Such alignment may, in some cases, align the knee in the outlier categories in a way that it might compromise correct load on the prosthesis posing a higher risk of failure. In 2012, Dossett et al. (6) reported the short-term radiological results of a randomized controlled trial comparing kinematically aligned total knee replacement (all performed using patience specific femoral and tibial cutting guide, OTISMED) and mechanically aligned TKR and in 2014 the same group of authors reported on the 2-year clinical results from this trial (24). A total of 88 patients ( 88 knees) were randomly allocated to undergo either kinematically aligned TKR using patient-specific guides, or mechanically aligned TKR using conventional instruments. In the last clinical review the authors concluded that the use of a kinematic alignment technique performed with patient-specific guides provided better pain relief and restored better function and range of movement than the mechanical alignment technique performed with conventional instruments. Another study has been published by Howell et al. (5) on functional outcomes of 214 manually kinematically aligned TKA for 3 alignment groups differentiated by alignment as in range $\left(-2.5^{\circ}\right.$ and $7.4^{\circ}$ valgus), varus (more than $-2.5^{\circ}$ ) and valgus (more than $7.4^{\circ}$ ). They found no significant difference according the Oxford Knee Score and Womac score.

\section{Discussion and conclusions}

Although neutral alignment is still considered the gold standard to obtain a long implant survivorship it is not sufficient so far to achieve a global pain free TKA during the whole arc of motion in a significant percentage of cases. Good clinical results depends on sound surgical technique and patient selection but it seems hard to believe that an alignment difference within $2^{\circ}-3^{\circ}$ between different alignment might explain poor patient outcome, increased implant stress and decreased survivorship. Encouraging results obtained with few reports about kinematically aligned TKA must be explained from a tridimensional point of view rather than bi-dimensional X-ray; a randomized trial showed that kinematically and mechanically aligned TKAs had similar limb and knee alignment but the tibial joint line was in more varus in the first group which is consistent with average $3^{\circ}$ varus tibial joint line of the general population. Such a kinematic approach is still under evaluation and larger studies are needed to establish which alignment method will promote the best clinical outcome after TKA. The concept of restoring the normal or prearthritic status when performing a TKA may be valid for a large number of patients but not all. Severe disease and severe preoperative metaphyseal malalignment makes difficult to apply a kinematic alignment without any risk to fall in the extreme outliers then increasing the risk of catastrophic failure. Concepts like ligament balancing and measured resection must always belong to the orthopaedic surgeon armamentarium in order to help him in the quest of the best possible result rather than blindly trusting one single method of alignment. 


\section{Acknowledgements}

None.

\section{Footnote}

Conflicts of Interest: The authors have no conflicts of interest to declare.

\section{References}

1. Noble PC, Conditt MA, Cook KF, et al. The John Insall Award: patient expectations affect satisfaction with total knee arthroplasty. Clin Orthop Relat Res 2006;452:35-43.

2. Bourne RB, Chesworth BM, Davis AM, et al. Patient satisfaction after total knee arthroplasty: who is satisfied and who is not? Clin Orthop Relat Res 2010;468:57-63.

3. Bellemans J, Colyn W, Vandenneucker H, et al. The Chitranjan Ranawat award: is neutral mechanical alignment normal for all patients? The concept of constitutional varus. Clin Orthop Relat Res 2012;470:45-53.

4. Eckhoff D, Hogan C, DiMatteo L, et al. Difference between the epicondylar and cylindrical axis of the knee. Clin Orthop Relat Res 2007;461:238-44.

5. Howell SM, Howell SJ, Kuznik KT, et al. Does a kinematically aligned total knee arthroplasty restore function without failure regardless of alignment category? Clin Orthop Relat Res 2013;471:1000-7.

6. Dossett HG, Swartz GJ, Estrada NA, et al. Kinematically versus mechanically aligned total knee arthroplasty. Orthopedics 2012;35:e160-9.

7. Hungerford DS, Krackow KA. Total joint arthroplasty of the knee. Clin Orthop Relat Res 1985;(192):23-33.

8. Pickering S, Armstrong D. Focus on alignment in total knee replacement. J Bone Joint Surg. 2012. Available online: http://www.boneandjoint.org.uk/content/focus/ alignment-total-knee-replacement.

9. Luo CF. Reference axes for reconstruction of the knee. Knee 2004;11:251-7.

10. Tang WM, Zhu YH, Chiu KY. Axial alignment of the lower extremity in Chinese adults. J Bone Joint Surg Am 2000;82-A:1603-8.

11. Hernigou P, Medevielle D, Debeyre J, et al. Proximal tibial osteotomy for osteoarthritis with varus deformity. A ten to thirteen-year follow-up study. J Bone Joint Surg Am 1987;69:332-54.

12. Sikorski JM. Alignment in total knee replacement. J Bone Joint Surg Br 2008;90:1121-7.
13. Scott W. Kinematic alignment in total knee arthroplasty. Insall \& Scott surgery of the knee. 5th ed. Churchill Livingstone: 2012.

14. Bellemans J, Carpentier K, Vandenneucker H, et al. The John Insall Award: Both morphotype and gender influence the shape of the knee in patients undergoing TKA. Clin Orthop Relat Res 2010;468:29-36.

15. Fahlman L, Sangeorzan E, Chheda N, et al. Older adults without radiographic knee osteoarthritis: knee alignment and knee range of motion. Clin Med Insights Arthritis Musculoskelet Disord 2014;7:1-11.

16. Insall JN, Binazzi R, Soudry M, et al. Total knee arthroplasty. Clin Orthop Relat Res 1985;(192):13-22.

17. Fang DM, Ritter MA, Davis KE. Coronal alignment in total knee arthroplasty: just how important is it? J Arthroplasty.2009;24:39-43.

18. Jeffery RS, Morris RW, Denham RA. Coronal alignment after total knee replacement. J Bone Joint Surg Br 1991;73:709-14.

19. Ritter MA, Davis KE, Meding JB, et al. The effect of alignment and BMI on failure of total knee replacement. J Bone Joint Surg Am 2011;93:1588-96.

20. Vanlommel L, Vanlommel J, Claes S, et al. Slight undercorrection following total knee arthroplasty results in superior clinical outcomes in varus knees. Knee Surg Sports Traumatol Arthrosc 2013;21:2325-30.

21. Parratte S, Pagnano MW, Trousdale RT, et al. Effect of postoperative mechanical axis alignment on the fifteenyear survival of modern, cemented total knee replacements. J Bone Joint Surg Am 2010;92:2143-9.

22. Hadi M, Barlow T, Ahmed I, et al. Does malalignment affect revision rate in total knee replacements: a systematic review of the literature. Springerplus 2015;4:835.

23. Abdel MP, Leopold SS. Editor's Spotlight/Take 5: Small improvements in mechanical axis alignment achieved with MRI versus CT-based patient-specific instruments in TKA: a randomized clinical trial. Clin Orthop Relat Res 2014;472:2909-12.

24. Dossett HG, Estrada NA, Swartz GJ, et al. A randomised controlled trial of kinematically and mechanically aligned total knee replacements: two-year clinical results. Bone Joint J 2014;96-B:907-13.

Cite this article as: Schiraldi M, Bonzanini G, Chirillo D, de Tullio V. Mechanical and kinematic alignment in total knee arthroplasty. Ann Transl Med 2016;4(7):130. doi: 10.21037/ atm.2016.03.31 